This paper is published in the open archive of Mid Sweden University

DIVA http://miun.diva-portal.org

with permission of the publisher

Citation for the peer-reviewed published paper:

Gärdlund L, Norgren M, Wågberg L, Marklund A. The use of polyelectrolyte complexes (PEC) as strength additives for different pulps used for production of fine paper. ; Nordic Pulp \& Paper Research Journal. $2007 ; 22(2): 210-216$.

URL to article at publishers site:

http://dx.doi.org/10.3183/NPPRJ-2007-22-02-p210-216 


\title{
The use of polyelectrolyte complexes (PEC) as strength additives for different pulps used for production of fine paper
}

\author{
Linda Gärdlund and Magnus Norgren, Mid Sweden University, Sundsvall, Sweden, Lars Wågberg, Royal Institute of Technology, KTH, Stockholm, \\ Sweden, Ann Marklund, M-Real Technology Center Örnsköldsvik, Sweden
}

KEYWORDS: Adsorption, Beating, Polyelectrolytes, Polyelectrolyte complexes, Tensile properties

SUMMARY: In this work the application of polyelectrolyte complexes (PEC) to different pulps, to improve the strength of papers produced from them, is compared with conventional pulp beating. The pulps chosen for the investigation were a fully bleached chemical hardwood pulp (HBK), a fully bleached chemical softwood pulp (SBK), and a peroxide-bleached chemithermomechanical pulp (BCTMP). The polyelectrolytes used were a polyamideamine epichlorohydrine condensate (PAE), traditionally used as a wet-strength additive, and carboxymethylcellulose. Since the prepared complexes had an anionic charge, they could only be used after fibre pre-treatment with the cationic PAE. Results indicate that the addition of $2 \%$ PAE and $2 \%$ PEC can improve all the measured tensile strength properties of the sheets as much as is commonly achieved by mechanically beating the pulps. In fact, for the chemical pulps, PEC addition at the chosen level produced better results than did traditional beating, since the improved tensile index and tensile energy absorption could be achieved without any significant deterioration in the light scattering coefficient of the papers produced. However, with the BCTMP a significant decrease in light scattering could be detected, especially following the addition of PEC.

Adding the chemicals (especially the PEC) increased the sheet density and this together with the increase in the tensile stiffness, following chemical addition, resulted in a constant bending stiffness of the sheets. However, based on the results it can be suggested that a combination of unbeaten HBK and BCTMP treated with PAE and PEC can be used to produce a strong paper with a high bending stiffness.

\section{ADDRESSES OF THE AUTHORS: Linda Gärdlund and Magnus Norgren: Mid Sweden University, Department of Natural Sciences, Fibre Science and Communication Network, FSCN, SE-85170 Sundsvall, Sweden. Lars Wågberg: Royal Institute of Technology, KTH, Department of Fibre and Polymer Technology, SE-100 44 Stockholm, Sweden. Ann Marklund: M-Real, Technology Center Örnsköldsvik, SE-891 80 Örn- sköldsvik, Sweden.}

Corresponding author: Lars Wågberg (wagberg@pmt.kth.se)

In the paper industry there is a constant strive to identify different approaches to improve the production economy for different paper grades with a maintained paper quality. Among these approaches the addition of fillers and cheaper fibre raw materials are perhaps the most common. However, these measures undoubtedly reduce paper strength, which must be counteracted by using wet-end strength additives (Reynolds, 1980; Lindström et al., 2005) or surface treating the paper with strength additives (Reynolds, 1980; Berg et al., 1988). Another way to maintain critical paper strength properties is to beat the fibres mechanically to different degrees, depending on the grade of paper to be produced (Wågberg and Annergren, 1997). This latter approach densifies the sheet and often reduces its bending stiffness, which is a severe problem, for example, in fine paper grades.

Traditionally, cationic starch has been the most costeffective strength additive (Reynolds, 1980), but over the past 5-10 years there has been a renewed effort to develop fibre treatments that improve paper strength without densifying the paper. In the recently developed BiPolarActivator (BPA) technique (Laine et al., 2000, 2002), carboxymethylcellulose is irreversibly deposited onto the pulp fibres, and results show that this treatment can produce improvements in the tensile strength properties of papers similar to those produced by traditional beating, but without densifying the sheet. Similar results have also been found in experiments in which the fibres have been treated using the polyelectrolyte multilayer (PEM) technique in which the fibres are consecutively treated with cationic and anionic polyelectrolytes to form multilayers on the fibres (Wågberg et al., 2002; Zheng et al., 2006). Recent investigations have also demonstrated that the effect of these multilayers is due to increased molecular contact in the contact zone between the fibres, increased work of adhesion in the areas of molecular contact, and an increased number of efficient fibre/fibre joints in the sheet (Eriksson et al., 2006). Industrially, this latter technique might be rather cumbersome, and a more versatile technique involving the premixing or in-situ combination of oppositely charged polyelectrolytes to form highly swollen polyelectrolyte complexes (PEC) (Gernandt et al., 2003; Gärdlund et al., 2003; Petzold et al., 1997; Hubbe, 2005) might be a more appealing fibre pre-treatment from a process integration point of view. It has been demonstrated that it is possible to tailor both the size and charge of the complexes by selecting the right mixing ratio, and to significantly improve the tensile strength properties of papers by a PEC treatment of the fibres before papermaking (Gärdlund et al., 2003).

These latter chemical modifications of the fibres (i.e., BPA, PEM, and PEC treatments) could all be used to reduce or entirely eliminate beating, but in practice, chemical additives are always used together with beating. This study therefore investigates how the effects of adding PEC, on different physical properties of the paper, can be compared with the effects achieved by beating the fibres. To establish how PEC could be used for a typical fine paper furnish three different types of pulps relevant for fine paper production were investigated: a fully bleached kraft hardwood pulp (HBK), a bleached chemi-thermomechanical pulp (BCTMP), and a fully bleached kraft softwood pulp (SBK). The PEC selected for the investigation comprised a mixture of a wet-strength resin (cationic polyamideamine epichlorohydrine - PAE) and an anionic carboxymethylcellulose (CMC), to enable comparison with earlier published results (Gärdlund et 
al., 2003). Since the complexes were anionically charged, they were combined with a single pre-treatment with the cationic PAE.

\section{Materials}

\section{Chemicals}

The polymers used to prepare the polyelectrolyte complexes (PEC) consisted of a polyamideamine epichlorohydrine (PAE) resin typically used as a wetstrength agent in the paper industry. It was obtained from Hercules AB (Helsingborg, Sweden); according to Gernandt et al. (2003), is has a broad molecular mass distribution with a weight-average molecular mass of approximately 1 million $\mathrm{g} / \mathrm{mol}$, as determined using static light scattering. The polymer was delivered as a $12 \%$ (by weight) solution and was used as received after dilution to the desired concentration with deionized water. The carboxymethylcellulose (CMC) was supplied by Metsa Specialty Chemicals AB (Finland ). According to the supplier, the weight-average molecular weight of this polymer is $300000 \mathrm{~g} / \mathrm{mol}$, but it was earlier determined to be $100000 \mathrm{~g} / \mathrm{mol}$, by using static light scattering (Gernandt et al., 2003).

In the polyelectrolyte titrations used to determine PAE adsorption, potassium polyvinyl sulphate from Wako Pure Chemicals (Japan) was used. To determine the anionic charge of the complexes and to determine PEC adsorption, polydimethyldiallyl ammonium chloride (polyDMDAAC) was used; this polymer was supplied by Ciba Specialty Chemicals(Bradford UK). The low-molecular-mass fraction of this polymer was removed by ultrafiltration and the weight-average molecular mass was determined by size exclusion chromatography to be $1.2 \cdot 10^{6} \mathrm{~g} / \mathrm{mol}$, according Swerin and Wågberg (1994). Analytical grade $\mathrm{NaOH}$ and $\mathrm{HCl}$ were used to adjust $\mathrm{pH}$ during the experiments.

\section{Pulps}

Three different pulps were used in the experiments. They were all delivered in dry form. Before use they were soaked in deionized water overnight and then reslushed according to ISO 5263-1:1997. The hardwood pulp, obtained from M-Real AB (Husum Mill, Sweden), was a fully bleached chemical pulp (HBK) mainly comprising birch; it was cooked to kappa 16 before being bleached in the sequence ODQPZP ( $2 \mathrm{~kg} / \mathrm{tp}$ ozone), to a final brightness of $90 \%$ ISO. The softwood pulp, obtained from M-Real AB (Husum Mill, Sweden), was a fully bleached chemical pulp (SBK) consisting of a mixture of spruce and pine; it was cooked to a kappa of 26 before being bleached in the sequence ODQEPDP, to a final brightness of $90 \%$ ISO. Finally, the bleached chemi-thermomechanical pulp (BCTMP), obtained from M-Real AB (Joutseno Mill, Finland), consisted of a mixture of aspen $(85 \%)$ and spruce $(15 \%)$ refined to a freeness of 110 CSF. The pulp was bleached with hydrogen peroxide to a final brightness of $81 \%$ ISO.

The charges of the specific pulps, used in the present investigation, were not determined but average values for the charges of these types of pulps; i.e., both the total charge, as determined by Katz et al. (1984) and the surface charge, as determined by polyelectrolyte adsorption (Wågberg et al., 1989) are summarized in Table 1 .

Table 1. Summary of the charges of the different pulps used in the investigation.

\begin{tabular}{lcc}
\hline Pulp & $\begin{array}{c}\text { Total charge } \\
{[\mu \mathrm{eq} / \mathrm{g}]}\end{array}$ & $\begin{array}{c}\text { Surface charge } \\
{[\mu \mathrm{eq} . / \mathrm{g}]}\end{array}$ \\
\hline HBK & $60-80$ & $18-34$ \\
SBK & $35-47$ & 12 \\
BCTMP & $160-200$ & $\mathrm{~N} / \mathrm{A}$ \\
\hline
\end{tabular}

\section{Methods}

\section{Polyelectrolyte complex preparation}

The CMC was dissolved in distilled water at a stock concentration of $0.5 \%$ and further diluted to a concentration of $0.22 \%$. The PAE was received as a concentrated solution; it was diluted to $0.5 \%$ and then further diluted to $0.22 \%$, also in distilled water. Before preparing the complex solutions, the $\mathrm{pH}$ of the PAE solution was adjusted to 7 with $0.01 \mathrm{M} \mathrm{NaOH}$; the $\mathrm{pH}$ of the $\mathrm{CMC}$ was approximately 6.8 after dilution and was not further adjusted.

The polyelectrolyte complexes were prepared in threelitre batches, by adding $1.5 \mathrm{~L}$ of the cationic polyelectrolyte solution to $1.5 \mathrm{~L}$ of the anionic polyelectrolyte solution, while stirring continuously. The addition was performed using a Metrohm 702 SM Titrino titrator (Herisau, Switzerland) at a dosage rate of $20 \mathrm{~mL} / \mathrm{min}$. The procedure for using this device to control the addition rate in complex formation followed an earlier described procedure (Dautzenberg 1997).

\section{Fibre treatment}

- Beating: the pulps were beaten in an Escher-Wyss laboratory refiner at four levels: $50-200 \mathrm{kWh} /$ tonne for both the hardwood (specific edge load, SEL, 1.0 $\mathrm{J} / \mathrm{m}$ ) and softwood pulps (SEL, 2.5 J/m) and 75-300 $\mathrm{kWh} / \mathrm{t}$ for the BCTMP pulp (SEL, $0.5 \mathrm{~J} / \mathrm{m}$ ).

- The pulp fibres were used untreated, treated with $2 \%$ PAE, and treated with PAE/PEC (also at a $2 \%$ addition level for each component). The dosage percentage is the dry weight of additive in relation to the amount of fibre material in the suspension. The stirring time after addition of chemical was $8 \mathrm{~min}$, and the adsorption was conducted at $\mathrm{pH} 7$. It should be added that an addition of $2 \%$ is large compared to the common additions of wet end cationic starch, which is usually below $1 \%$. However, the ambition with the present work was to critically test the potential of the addition of complexes and therefore this level of addition was chosen.

\section{Sheet preparation}

The paper sheets (grammage, $80 \mathrm{~g} / \mathrm{m}^{2}$ ) were formed in a Rapid Köthen sheet former from Paper Testing Instruments (Pettenbach, Austria) from a $0.3 \%$ fibre suspension. The sheets were dried on site at $94^{\circ} \mathrm{C}$ at a pressure of $90 \mathrm{kPa}$. Afterwards, the formed paper sheets were cured at $105^{\circ} \mathrm{C}$ for $20 \mathrm{~min}$ in an oven, to allow for full reaction between the PAE and the fibres. 


\section{Determination of the adsorbed amount of additive}

In this part of the investigation unbeaten HBK, SBK, and BCTMP fibres were used. To determine the amount of PAE adsorbed onto the fibres, polyelectrolyte titration (Terayama, 1952; Wågberg, 1989) was used. To detect the point of charge reversal during the titration, a Mütek PCD 03 a streaming current detector (Herrsching, Germany) was used (Walker et al., 1996).

Before adsorbing the polyelectrolyte complexes onto the fibres, they were cationized by adding $2 \%$ (by weight) of the PAE solution and then stirring for $8 \mathrm{~min}$; after this, the pulp suspension was filtered and re-diluted three times with deionized water. Then the PEC solution was added to the fibres, and after filtration, the adsorbed amount was determined using polyelectrolyte titration. The adsorption time was 15 min for both the individual PAE adsorption isotherm and the complex isotherm, and a constant $\mathrm{pH}$ of 7 was maintained. This means that the adsorption procedure is a bit different from the sheet preparation procedure.

\section{Sheet testing}

The laboratory sheets were tested using the standard methods presented in Table 2.

The testing of the sheets is based on repeated measurements of 10 samples and the coefficients of variation of the measured values are below $5 \%$.

Table 2. Summary of the methods used to characterize the properties of the prepared laboratory sheets.

\begin{tabular}{ll}
\hline Analysis & Method \\
\hline Basis weight, $\mathrm{g} / \mathrm{m}^{2}$ & SCAN-P 6:75 \\
Bulk thickness, $\mu \mathrm{m}$ & SCAN-P 7:96 \\
Bulk density, $\mathrm{kg} / \mathrm{m}^{3}$ & SCAN-P 7:96 \\
Tensile index, Nm/g & SCAN-P 67:93 \\
Tensile stiffness index, MNm/kg & SCAN-P 67:93 \\
Tensile energy absorption index, J/ $\mathrm{m}^{2}$ & SCAN-P 67:93 \\
Elongation, $\%$ & SCAN-P 67:93 \\
E-modulus, GPa & SCAN-P 67:93 \\
Optical properties & SCAN-P 93 \\
\hline
\end{tabular}

\section{Results}

\section{Mechanical properties of papers made from the treated fibres}

The three different pulps, beaten to different degrees, were treated with PAE and PAE/PEC to investigate whether PEC treatment could offer an alternative to mechanically beating the fibres. In the method used here (i.e., anionic PEC treatment), it is necessary to combine the PEC treatment with a pretreatment with a cationic polymer to cause a charge reversal of the furnish. For a fair comparison it was decided to use PAE for charge reversal. It has to be stressed, however, that there are cheaper alternatives to such a pre-treatment (that, however, is a matter for future study).

After drying and conditioning the papers, their tensile strength properties were evaluated; the following summarizes these investigations. As seen in Figs 1 a-c, both PAE treatment and PAE/PEC treatment of the HBK pulp did significantly change the tensile strength properties of the paper formed. Comparing the results of chemical
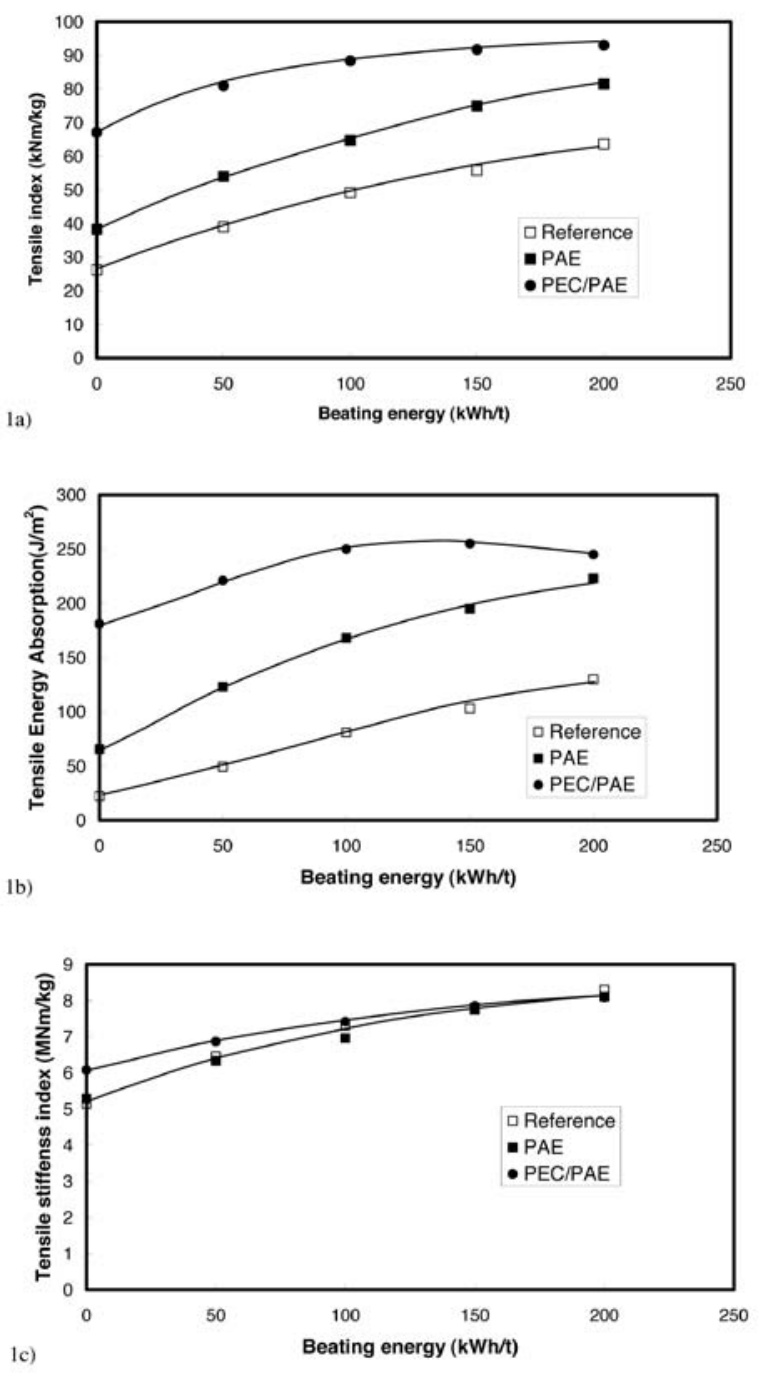

Fig 1. Summary of the tensile strength properties achieved with HBK fibres subjected to chemical treatment (PAE or PAE/PEC), mechanical beating, or a combination of both. a) Tensile index, b) Tensile energy absorption, and c) Tensile stiffness index. In the experiments with PAE, PAE was added to $2 \%$ of the dry weight of the fibres in suspension; in the complex treatments, fibres were pre-saturated with $2 \%$ PAE before the addition of PEC to $2 \%$ of the dry weight of the fibres. The lines in the figure merely serve to guide the eye.

treatment plus no or different degrees of beating with the results of only beating the fibres (labelled "Reference" in the figure), it is clear that the addition of the chemicals could basically replace the beating.

Fig $1 b$ also indicates large increases in the tensile energy absorption of the sheets, due to the combined increase in tensile index and strain at break of the sheets. From Fig $1 \mathrm{c}$ it is also clear that the chemicals could not induce the same increases in the tensile stiffness index of the sheets as beating could, as has also been found with other types of chemical additives (Lindström et al., 2005). With higher degrees of beating, the chemical additives in fact had no effect on the tensile stiffness index. It can be argued that at these rather high tensile stiffness levels, the full potential of this fibre type has already been achieved, and that to increase tensile stiffness still further the fibre wall would have to be strengthened, for example, by chemical cross-linking (Caulfield, 1994). As seen in Figs $2 a-c$ and $3 a-c$, similar results were achieved for the SBK and BCTMP pulps. 


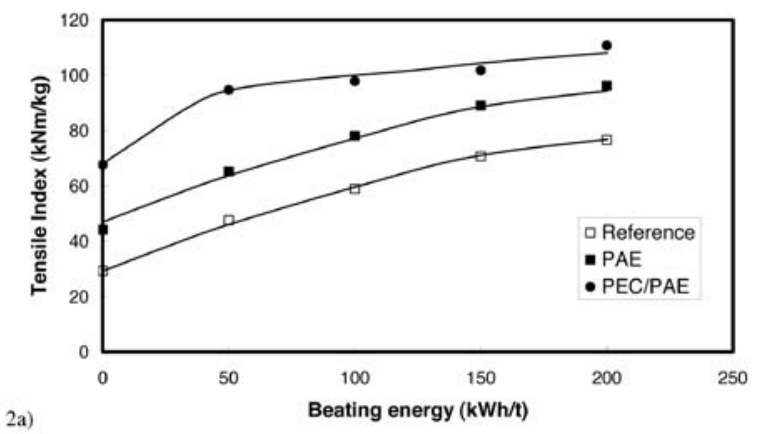

2a)
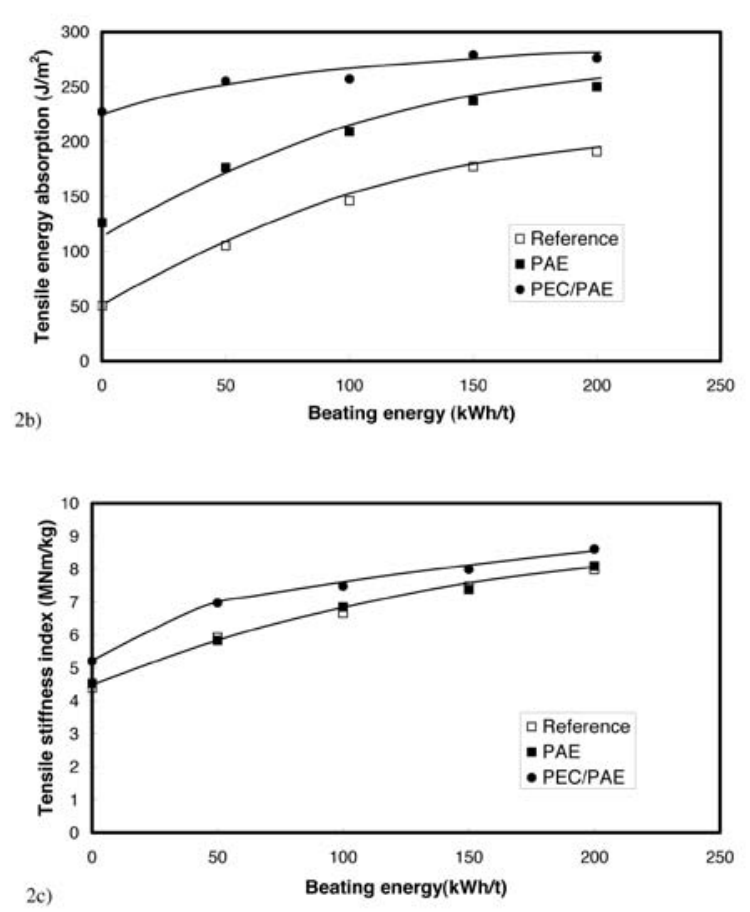

Fig 2. Summary of the tensile strength properties achieved with SBK fibres subjected to chemical treatment (PAE or PAE/PEC), mechanical beating, or a combination of both. a) Tensile index, b) Tensile energy absorption, and c) Tensile stiffness index. In the experiments with PAE, PAE was added to $2 \%$ of the dry weight of the fibres in suspension; in the complex treatments, fibres were pre-saturated with $2 \%$ PAE before the addition of PEC to $2 \%$ of the dry weight of the fibres. The lines in the figure merely serve to guide the eye.

Despite the general similarities, there were some specific differences between the results for the different pulps. First, the addition of PAE/PEC increased the tensile stiffness index over the entire beating range for both the SBK and BCTMP pulps. For BCTMP the improvement was approximately $15 \%$, whereas for SBK it was approximately $20 \%$ at a beating energy of $50 \mathrm{kWh} / \mathrm{t}$. Second, it is clear that the tensile properties of BCTMP sheets could essentially not be improved by mechanical beating, in line with earlier results and expectations (Höglund, 2006); the treatment was nonetheless included in the present study to allow for full comparison of the different pulps. It should also be stressed that PAE/PEC treatment of BCTMP resulted in a tensile index similar to and a tensile energy absorption only $40 \%$ under those of the non-chemically treated SBK at a beating energy of $200 \mathrm{kWh} / \mathrm{t}$.

\section{Polymer and PEC adsorption}

Considering the positive effects of the chemical treatments on the mechanical properties of papers made
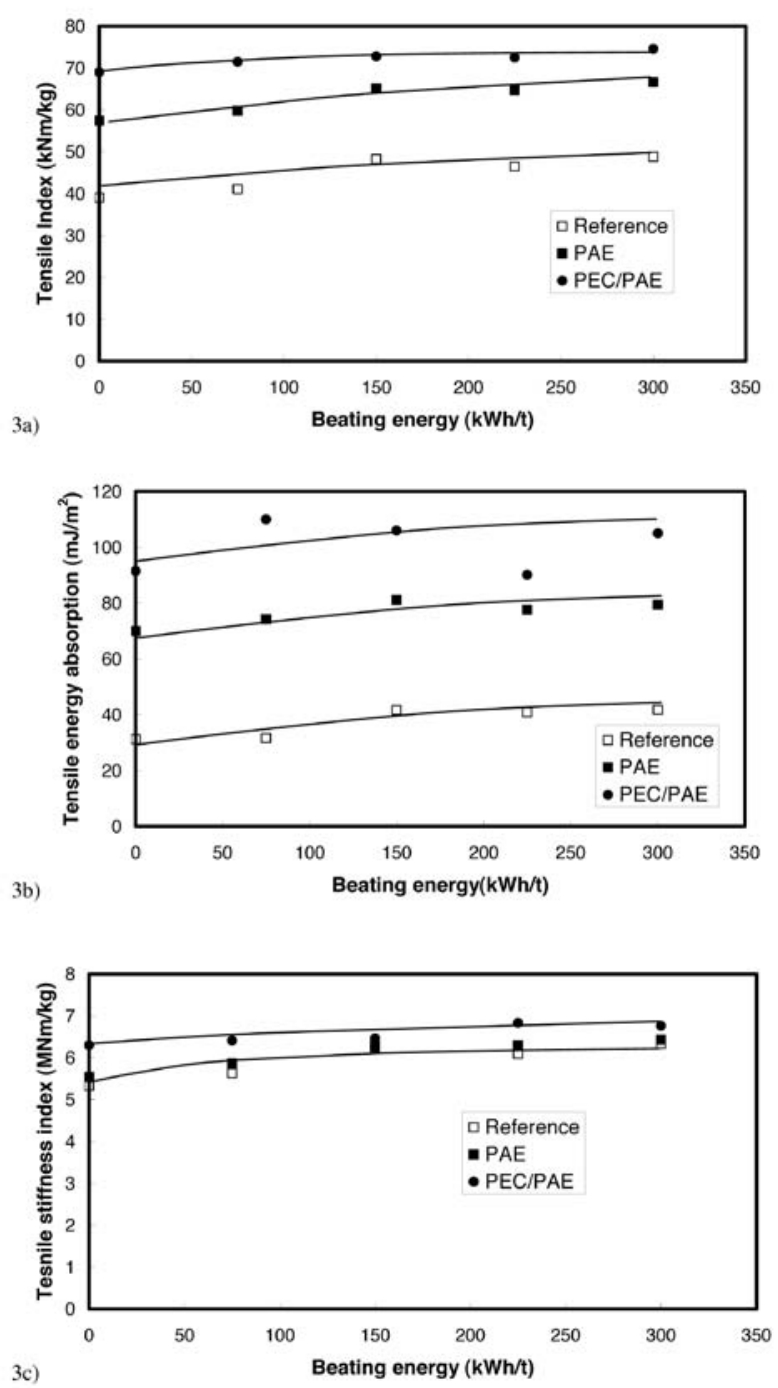

Fig 3. Summary of the tensile strength properties achieved with BCTMP fibres subjected to chemical treatment (PAE or PAE/PEC), mechanical beating, or a combination of both. a) Tensile index, b) Tensile energy absorption, and c) Tensile stiffness index. In the experiments with PAE, PAE was added to $2 \%$ of the dry weight of the fibres in suspension; in the complex treatments, fibres were presaturated with $2 \%$ PAE before the addition of PEC to $2 \%$ of the dry weight of the fibres. The lines in the figure merely serve to guide the eye.

from beaten and unbeaten fibres, it is important to determine the amounts of the chemicals adsorbed, to determine the full potential of the chemical treatments. Therefore, unbeaten fibres treated with either PAE or PAE/PEC were subjected to adsorption measurements; these results are presented in Fig 4 (PAE) and Fig 5 (PEC on fibres pre-treated with PAE).

As seen in Fig 4, the hardwood and the softwood pulps displayed similar saturation adsorption values for the PAE. There was, however, a tendency for higher adsorption onto the hardwood fibres, which is understandable considering the higher charge of this pulp due to its higher content of charged glucuronoxylan. It is also clear that much more PAE was adsorbed onto BCTMP than onto the bleached chemical pulps. This was probably due to the higher charge of this pulp in combination with its higher fines concentration, which makes a larger area available to the PAE molecules.

For the complexes the situation was quite different. As seen in Fig 5, the hardwood pulp had the highest 


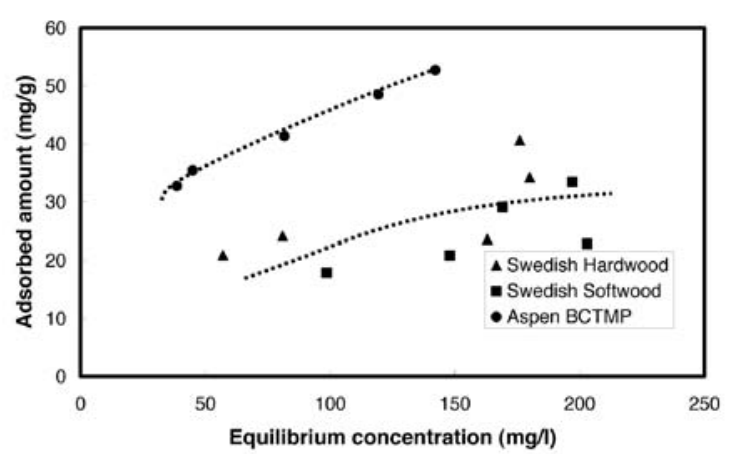

Fig 4. Adsorption of PAE onto the different unbeaten pulps investigated here. The experiments were conducted in deionized water and at $\mathrm{pH}$ 7. The lines in the figure merely serve to guide the eye.

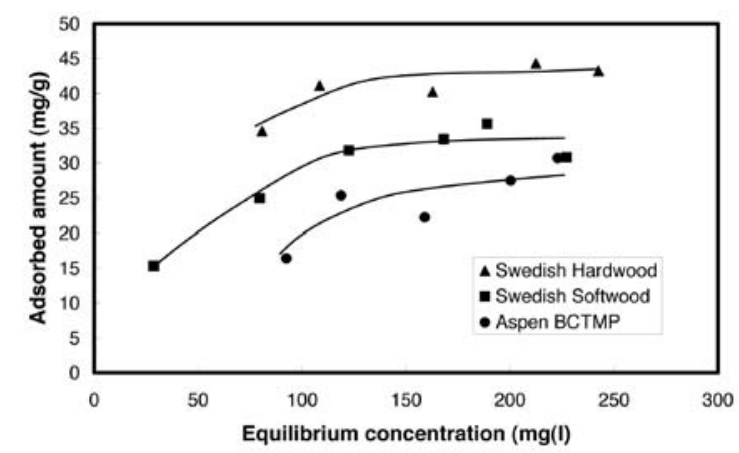

Fig. 5 Adsorption of PEC onto the different unbeaten pulps investigated here. The experiments were conducted in deionized water and at $\mathrm{pH} 7$; the fibres were presaturated with $2 \%$ PAE to reverse the charge, to allow for adsorption of the anionic polyelectrolyte complexes and the fibres were thoroughly rinsed between the adsorption of PAE and adsorption of the complexes. The lines in the figure merely serve to guide the eye.

adsorption; the softwood pulp had lower adsorption and BCTMP the lowest. This could be because the addition of PAE was insufficient to completely recharge the BCTMP fibres. This will be further discussed later.

\section{Discussion}

\section{Influence of the chemicals on interaction between the fibres}

The results presented in Figs 1-3 indicate that there is great potential for PEC application to replace much of the mechanical beating of the fibres. However, in practice there are other necessary conditions: the cost of the chemicals, the influence of the chemicals on the papermaking process, and the effects of the chemicals on, for example, the optical properties of papers made from the treated fibres. It was thus essential to determine how the optical properties, i.e., the light scattering coefficient, of the papers change with beating versus with the addition of chemicals. As seen in Figs $6 a-c$, the chemicals had similar and moderate effects on the sheets made of the chemical pulps, but significantly reduced the light scattering of the BCTMP sheets.

For the chemical pulps, the treatments greatly increased the tensile index without significantly reducing the light scattering coefficient of the papers. This indicates that the added chemicals improved the contacts in the contact zone between the fibres. It is obviously difficult, if not impossible, to draw any molecular-level
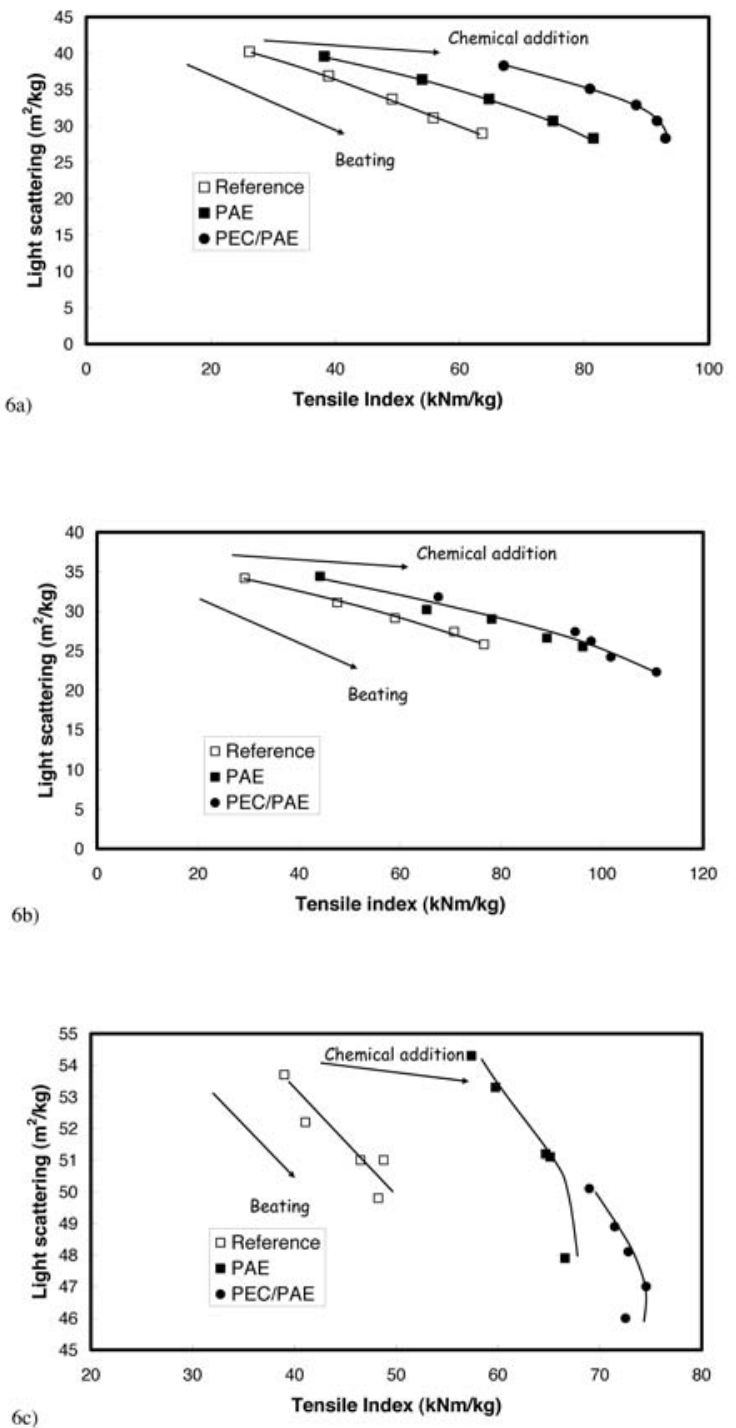

Fig 6. Light scattering as a function of tensile index for sheets subjected to both chemical treatment (PAE, PAE/PEC) and mechanical beating. a) HBK, b) SBK, and c) BCTMP. The lines in the figure merely serve to guide the eye.

conclusions from sheet testing. However, comparing these data to the results of a recent similar investigation (Eriksson et al., 2006) suggests that both the PAE and PAE/PEC treatments improved both the molecular contact area between fibres and possibly the molecular work of adhesion between fibre surfaces (considering the reactivity of the $\mathrm{PAE}$ and $\mathrm{PAE} / \mathrm{CMC}$ system), without changing the light scattering coefficient of the sheets. This would obviously be very advantageous for the applicability of the PEC in practice, but it must be remembered that filler particles dominate light scattering in a typical fine paper, so further testing with filler also present is necessary to clarify the full effect of PEC addition on the optical properties of paper.

As mentioned earlier, the effects of both the PAE and PAE/PEC treatments on BCTMP differed significantly from their effects on the chemical pulps. The effect of the PAE/PEC treatment on light scattering was also much greater than that of the single addition of PAE, in which significant effects were detected only in the fibres beaten the most. Overall, PEC addition decreased the light scattering by approximately four units compared with just beating the fibres. This decrease could be caused by 


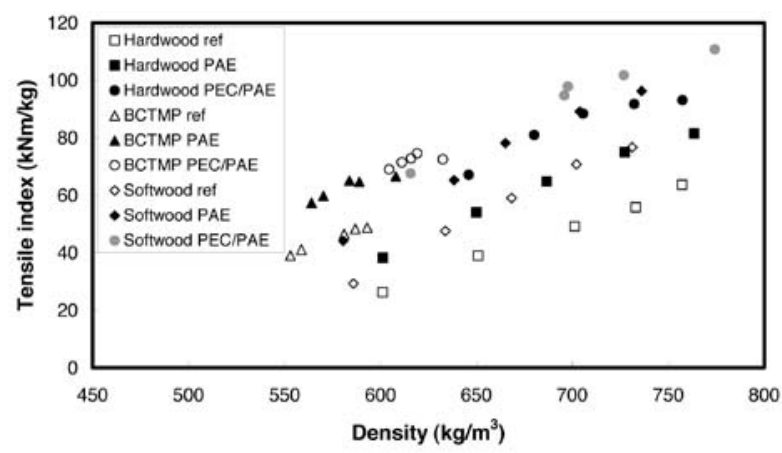

Fig 7. Summary of the relationship between tensile index and sheet density for all pulps studied in this investigation.

both increased consolidation between the BCTMP fibres and flocculation of the fines material, since both these will reduce the light scattering. Increased consolidation between fibres caused by PEM treatment of BCTMP has recently been reported (Pettersson et al., 2006), and much of the decreased light scattering arising from the PEC addition could be due to better contact between the BCTMP fibres, in particular, in areas available for light scattering. Since the decrease in light scattering is approximately the same regardless of the amount of fines in the furnish (i.e., the degree of beating), the major cause of the decreased light scattering may have been higher consolidation between the furnish components rather than fines flocculation. However, there might have been some agglomeration of the fines, and the influence of this on paper properties remains to be tested.

Notably, the highly swollen macroscopic PEC particles are what is needed to bridge the surfaces of the BCTMP fibres, thus causing a larger reduction in light scattering; this is suggested by the large difference in terms of effect on light scattering between the PAE and PEC additions.

When considering paper consolidation, it is also important to clarify how the different additives increase the density of sheets made from the different pulps; Fig 7 presents a summary of the correlation between tensile index and sheet density for all the pulps studied here.

The general trend that can be extracted from these data is that saturation of the fibres with PAE essentially increases the tensile index without affecting sheet density, whereas PEC addition leads to significant densification. Once again, this is in line with recently published findings regarding the PEM treatment of fibres (Pettersson et al., 2006); obviously, this densification is a drawback for the bending stiffness of the sheets.

\section{Combining different pulp treatments to prepare a typical fine paper furnish of optimum performance}

When considering the application of the results discussed so far, it is important to remember that the addition of different types of pulps has different purposes in a typical fine paper furnish, depending on the desired properties of the final paper. The major reason for using long-fibre pulp is to achieve sufficient strength (i.e., tensile stiffness and tensile strength), while short-fibre pulp is used to achieve better light scattering and formation with sufficient sheet strength. Finally, BCTMP can be added to increase the sheet bulk, to produce a final paper of high bending stiffness. It was thus considered of interest to

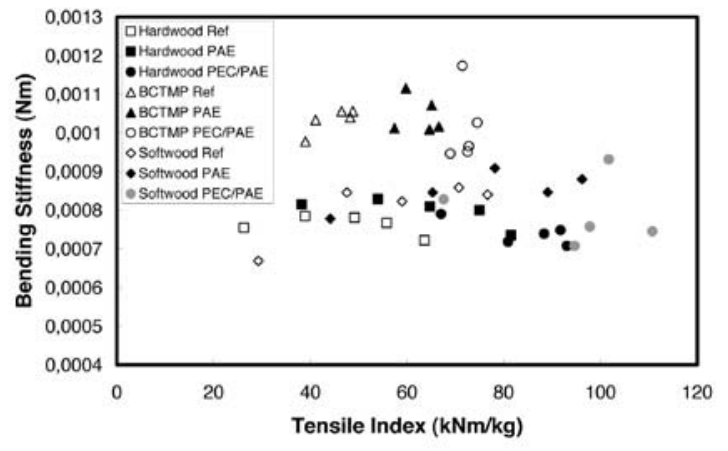

Fig 8. Summary of the relationship between bending stiffness and tensile index for all pulps studied here.

compare papers made from the different pulps and with the different chemical treatments, with respect to bending stiffness and tensile strength. Such a comparison is presented in Fig 8 , in which the calculated bending stiffness (i.e., $\mathrm{Et}^{3} / 12$, where $E$ is the elastic modulus, $\left[\mathrm{N} / \mathrm{m}^{2}\right]$, and $\mathrm{t}$ the thickness of the sheet, [m]) is presented as a function of the tensile index for all the pulps studied here.

From Fig 8 it is clear that the addition of chemicals greatly improved the tensile index; however, due to the rather small effect on the tensile stiffness (seen in Figs 1-3) and densification (seen in Fig 7) of the sheets, the treatment had basically no positive effect on the bending stiffness. However, as can be seen, BCTMP sheets still had a much higher bending stiffness than did chemical pulp sheets. Considering the large improvements of the tensile strength properties of the hardwood pulp and of the BCTMP following PEC addition, the long-fibre pulp could possibly be totally replaced with PEC-treated hardwood pulp and PEC-treated BCTMP in a typical fine paper furnish. Furthermore, mechanical treatment of the hardwood pulp could possibly be avoided as long as the desired surface smoothness of the paper can be achieved. These measures should improve the runnability of the paper machine by producing less fines, and improve the dimensional stability of the paper by reducing or completely eliminating the beating. Of course, this is only a hypothesis based on the present results, which remains to be tested.

\section{Adsorption of PEC onto PAE pre-saturated unbeaten fibres}

As mentioned earlier, the adsorption of PAE followed expected trends concerning the fibre charge and the amount of fines in the pulps. However, the adsorption of PEC onto pulps pre-saturated with PAE did not follow the trend established by the initial adsorption, indicating that the PAE was not equally available on the different pulps. There are several possible explanations for this behaviour. Regarding the difference between the mechanical and chemical pulps, the strength of interaction between the more highly charged BCTMP and the PAE is so high that the PAE will display a very flat conformation on the BCTMP surface; this will result in a lower availability to the PEC added in the second step. The agglomeration of fines will also reduce the availability of the adsorbed PAE to the added PEC. The adsorption of cationic polyacrylamide onto aggregated polystyrene latex was earlier found to display such behaviour, and the magnitude of the effect can be significant (Tanaka et al., 
1990). The relatively low adsorption of PEC onto the presaturated BCTMP could also be because the fibres were not totally recharged by the PAE, considering the rather high slope of the adsorption isotherm of PAE on BCTMP; this possibility should be tested in future trials.

The difference found between the softwood and hardwood pulps is more difficult to explain. Possibly, the morphology of the hardwood pulp, characterized by open vessel cells or long slender libriform cells, is advantageous for the availability of the pre-adsorbed PAE to the sequentially added PEC given a certain amount of preadsorbed PAE. In the softwood pulps, a large fraction of the PAE may be adsorbed inside the lumen or in pores in the fibres that are inaccessible to larger PEC particles, the longest dimension of which is $20-100 \mathrm{~nm}$ (Gernandt et al., 2003). Since the differences between the pulps are considerable in this respect, and since to the best of our knowledge this effect has not been demonstrated before, future trials are needed.

\section{Conclusions}

This investigation demonstrates that in sheets made of a hardwood bleached kraft pulp, a softwood bleached kraft pulp, and an aspen BCTMP, the addition of polyelectrolyte complexes can improve the tensile properties even more than mechanical beating can. Results also indicate that different combinations of beating and chemical additives can be used to produce even stronger sheets. The tensile index and tensile energy absorption are improved greatly by PEC addition, whereas the tensile stiffness index is improved to a considerably smaller extent. PEC addition also significantly densifies the sheets, and together with the relatively small increases in tensile stiffness caused by PEC addition, this means that the bending stiffness is basically unaffected by PEC addition. However, the BCTMP still has a much higher bending stiffness than do the bleached chemical pulps, so the results indicate that adding $\mathrm{PEC}$ to a combination of unbeaten BCTMP and unbeaten hardwood pulp would be sufficient to produce a fibre furnish with a sufficient strength potential for production of a typical fine paper.

The results of adsorbing both PAE and PEC onto pulps pre-saturated with PAE indicate that the hardwood pulps can adsorb more PEC despite adsorbing less PAE than the BCTMP pulp can. This indicates that the hardwood pulps seem especially suited for PEC treatment; there are unfortunately no unambiguous explanations for these latter results.

\section{Literature}

Berg, N.-0., Glittenberg, D. and Weinbach, H. (1988): Surface treatment of paper in size press and film press, Papier 42(10A),V40-V47.

Caulfield, D.F. (1994): Ester crosslinking to improve wet performance of paper using multifunctional carboxylic acids, butanetetracarboxylic and citric acid, Tappi J. 77(3) 205-212.

Dautzenberg, H. (1997): Polyelectrolyte complex formation in highly aggregating systems. 1. Effects of salt: Polyelectrolyte complex formation in the presence of salt, Macromolecules 30, 7810-7815.

Eriksson, M., Torgnysdotter, A. and Wågberg, L. (2006): Surface modification of wood fibers using the polyelectrolyte multilayer technique: Effects on fiber joint and paper strength properties, Industrial \& Engineering Chemistry Res. 45(15) 5279-5286

Gernandt, R., Wågberg, L., Gärdlund, L. and Dauzenberg, H. (2003): Polyelectrolyte complexes for surface modification of wood fibres. Part I: Preparation and characterization of complexes for dry and wet strength improvement of fibres, Colloids and Surfaces A. Physiochem. Eng. Aspects, 213, 15-25.

Gärdlund, L., Wågberg, L. and Gernandt, R. (2003): Polyelectrolyte complexes for surface modification of wood fibres. Part II: Influence of complexes on wet and dry strength of paper, Colloids Surf. 218(1-3), 137-149.

Hubbe, M.A. (2005): Dry-strength development by deposition of polyelectrolyte complexes onto non-bonding glass fibres, J. Pulp Paper Sci. 31(4) 159-166.

Höglund, H. (2006): Private communication (hans.hoglund@miun.se)

Katz, S., Beatson, R. and Scallan, A. (1984): The determination of strong and weak acidic groups in sulfite pulps, Svensk Papperstidning, 87(6), 48-53.

Laine, J., Lindström, T., Glad-Nordmark, G. and Risinger, G. (2000): Studies on topochemical modification of cellulose fibres. Part 1, Nord. Pulp Paper Res. J. 15(5) 520-526.

Laine, J., Lindström, T., Glad-Nordmark, G. and Risinger, G. (2002): Studies on topochemical modification of cellulosic fibres. Part 2: The effect of carboxymethyl cellulose attachment on fibre swelling and paper strength, Nord. Pulp Paper Res. J. 17(1) 50-56.

Lindström, T., Wågberg, L. and Larsson, T. (2005): On the nature of joint strength in paper - A review of dry and wet strength resins used in paper manufacturing, In: l'Anson, S. J. (ed.), Advances in Paper Science and Technology, The Pulp and Paper Fundamental Res. Soc., UK, ISBN 0954527232, pp. 457-562.

Pettersson, G., Höglund, H. and Wågberg, L. (2006): The use of polyelectrolyte multilayers of cationic starch and CMC to enhance strength properties of papers from mixtures of unbleached chemical pulp and CTMP. Part 1, Nord. Pulp Paper Res. J. 21(1) 115-121.

Petzold, G., Schwarz, S. and Buchhammer, H.M. (1997): A very effective method for the cationic modification of cellulose, Angewandte Makromolekulare Chemie 253, 1-15.

Reynolds, W.F. (1980): Dry Strength Additives, Tappi Press, ISBN 0898520444 , Atlanta, Ga, USA.

Swerin, A. and Wågberg, L. (1994): Use of HPLC methodology to characterize cationic polymers used in the paper industry, Nord. Pulp and Paper Res. J., 9(1) 18. Tanaka, H., Ödberg, L., Wågberg, L. and Lindström, T. (1990): Adsorption of cationic polyacrylamides onto monodisperse polystyrene latices and cellulose fiber: Effect of molecular weight and charge density of cationic polyacrylamides, J. Colloid Interface Sci. 134(1) 219-228.

Terayama, H. (1952): Method of colloid titration (a new titration between polymer ions), J. Polym. Sci. 8(2), 243-253.

Walker, C., Kirby, J. and Dentel, S. (1996): The streaming current detector: A quantitative model, J. Colloid Interface Sci. 182, 71-81.

Wågberg, L., Ödberg, L. and Glad-Nordmark, G. (1989): Charge determinations of porous substrates by polyelectrolyte adsorption. Part 1: Carboxymethylated, bleached, cellulosic fibres, Nord. Pulp Pap. Res. J. 4(2) 71-76.

Wågberg, L. and Annergren, G. (1997): Physicochemical characterization of papermaking fibres, In: Baker, C. F. (ed.), Fundamentals of Papermaking Materials, Pira Intern., UK, ISBN 1858022096, pp. 1-82.

Wågberg, L., Forsberg, S., Johansson, A. and Juntti, P. (2002): Engineering of fibre surface properties by application of the polyelectrolyte multilayer concept. Part 1: Modification of paper strength, J. Pulp Paper Sci. 28(7), 222-228.

Zheng, Z., McDonald, J., Khillan, R., Su, Y., Shutava, T., Grozdits, G. and Lvov, Y.M. (2006): Layer by layer nanocoating of lignocellulose fibres for enhanced paper properties, J. Nanoscience Nanotechnology, 6, 1-9. 